\title{
Evaluation of a sorbitol dehydrogenase inhibitor on diabetic peripheral nerve metabolism: a prevention study
}

\author{
I. G.Obrosova ${ }^{1}$, L. Fathallah ${ }^{1}$, H.J. Lang ${ }^{2}$, D. A. Greene ${ }^{1}$ \\ ${ }^{1}$ Division of Endocrinology and Metabolism, Department of Internal Medicine, University of Michigan Medical Center, \\ Ann Arbor, Michigan, USA \\ ${ }^{2}$ Hoechst Marion Roussel Deutschland GmbH, Chemical Research Frankfurt, Frankfurt/Main, Germany
}

\section{Abstract}

Aims/hypothesis. Studies of the role of sorbitol dehydrogenase in nerve functional deficits induced by diabetes reported contradictory results. We evaluated whether sorbitol dehydrogenase inhibition reduces metabolic abnormalities and enhances oxidative stress characteristic of experimental diabetic neuropathy.

Methods. Control and streptozotocin-diabetic rats were treated with or without sorbitol dehydrogenase inhibitor (SDI)-157 (100 mg $\mathrm{kg}^{-1} \cdot \mathrm{day}^{-1}$, in the drinking water, for 3 weeks). Sciatic nerve free mitochondrial (cristae and matrix) and cytosolic NAD ${ }^{+}$: $\mathrm{NADH}$ ratios were calculated from the $\beta$-hydroxybutyrate, glutamate and lactate dehydrogenase systems. Concentrations of metabolites, e.g. sorbitol pathway intermediates and variables of energy state were measured in individual nerves spectrofluorometrically by enzymatic procedures.

Results. The flux through sorbitol dehydrogenase (manifested by nerve fructose concentrations) was inhibited by $53 \%$ and $74 \%$ in control and diabetic rats treated with SDI compared with untreated control and diabetic groups. Free $\mathrm{NAD}^{+}: \mathrm{NADH}$ ratios in mi- tochondrial cristae, matrix and cytosol were decreased in diabetic rats compared with controls and reduction in either of the three variables was not prevented by sorbitol dehydrogenase inhibitor. Phosphocreatine concentrations and phosphocreatine:creatine ratios were decreased in diabetic rats compared with controls and were further reduced by the inhibitor. Malondialdehyde plus 4-hydroxyalkenals concentration was increased and reduced gluthathione concentration was reduced in diabetic rats compared with the control group, and changes in both variables were further exacerbated by sorbitol dehydrogenase inhibitor. Neither NAD-redox and energy states nor lipid aldehyde and reduced gluthathione concentrations were affected by treatment with the inhibitor in control rats.

Conclusion/interpretation. Inhibition of sorbitol dehydrogenase does not offer an effective approach for prevention of oxidation and metabolic imbalances in the peripheral nerve that is induced by diabetes and is adverse rather than beneficial. [Diabetologia (1999) 42: 1187-1194]
Received: 19 April 1999 and in revised form: 28 May 1999

Corresponding author: Dr. I. Obrosova, Division of Endocrinology and Metabolism, Department of Internal Medicine, University of Michigan Medical Center, 1150 West Medical Center Drive, MSRB II, Rm 5570, Ann Arbor, Michigan 48109-0354, USA

Abbreviations: SDH, sorbitol dehydrogenase; SDI, sorbitol dehydrogenase inhibitor; GSH, reduced glutathione.
Numerous studies in experimental models of diabetes [1-5] as well as a few clinical trials [6-9] have shown a reduction of peripheral nerve conduction deficit and morphological abnormalities by structurally different aldose reductase inhibitors thus implicating increased activity of the sorbitol pathway in the pathogenesis of diabetic neuropathy. The mechanisms leading from increased sorbitol pathway activity to complex functional, metabolic and morphological changes in the peripheral nervous system in diabetes are not com- 
pletely understood. A number of reports $[1,2,4$, 10-14] link vascular [4, 15-20], metabolic [21-24] and neurotrophic [25-27] imbalances that are induced by diabetes to the activity of the first half of the sorbitol pathway, aldose reductase. Some investigators [28, 29] have hypothesized that adverse consequences of increased sorbitol pathway activity in the diabetic nerve are not due to aldose reductase activation itself, but are related to the second half of the sorbitol pathway, sorbitol dehydrogenase (SDH). According to the concept of "diabetic pseudohypoxia" [28], the increased flux through SDH accounts for NAD-redox imbalances (increase in NADH:NAD ${ }^{+}$ ratio) related to diabetes and constitutes a universal mechanism for development of diabetic complications including neuropathy, retinopathy and nephropathy. Studies of the role for SDH in diabetic complications using SDH inhibitors (SDIs) $[4,30,31]$ as well as a SDH-deficient mouse model [32] resulted in contradictory findings. Some groups [29-31] have reported that SDIs reduce nerve conduction deficit induced by diabetes. Others failed to find improved nerve conduction in diabetic rats treated with SDI compared with an untreated diabetic group [4] or in SDH-deficient diabetic mice compared with diabetic mice with normal SDH levels [32].

For a better understanding of biochemical mechanisms underlying diabetic neuropathy, we explored metabolic effects associated with SDH inhibition in diabetic peripheral nerve. Metabolic changes in nerve vasculature, with resulting decrease in nerve blood flow and endoneurial hypoxia, have a key role in nerve conduction slowing in short-term diabetes [4, 15-20]. Metabolic imbalances in the neural tissues, closely associated with impaired neurotrophism [13, 25-27, 33] and neurotransmission [34-36], contribute to Schwann cell injury $[37,38]$ and axonopathy $[39$, 40]. These abnormalities which develop gradually and become manifest in long-standing diabetes [38, 39] exacerbate nerve functional deficits acquired in the initial phase of diabetic neuropathy. The importance of sorbitol pathway-linked metabolic imbalances in neural structures is illustrated by findings [41] of a large increase in neuroaxonal dystrophy with SDH inhibition by the dose of SDI not affecting nerve blood flow in the diabetic model of lesser duration [4]. Out of a variety of metabolic variables, we have selected nerve energy status and indices of oxidative stress. Nerve energy status is closely associated with the redox state of free motochondrial and cytosolic NAD-couples, active transport and biosynthetic activity and directly correlates with nerve conduction [42-44]. Oxidative stress has been implicated in the impairment of neurotrophic support and nerve dysfunction in diabetes $[26,45]$. We compared our variables in control and diabetic rats treated with or without SDI. We used a prevention approach giving SDI in the drinking water i. e. conditions which, according to some investigators [31], are essential to show the effects of SDIs. The metabolic effects of SDIs (except those on the sorbitol pathway intermediates $[4,30])$ in the diabetic nerve have only been studied in nonsteady state endoneurial incubations in vitro [29] in high glucose medium. This does not exactly mimic the metabolic environment in diabetic tissues.

\section{Materials and methods}

The experiments were done in accordance with regulations specified by The Guiding Principles in the Care and Use of Animals (DHEW Publication, NIH 80-23) and the University of Michigan Protocol for Animal Studies.

Animals. Barrier-sustained, Caesarean-delivered male Wistar rats (Charles River, Wilmington, Mass., USA), body weight 250-300 g, were fed a standard rat chow diet (ICN Biomedicals, Cleveland, Ohio, USA) and had access to water ad libitum. Diabetes was induced by a single intraperitoneal injection of streptozotocin (Upjohn, Kalamazoo, Mich., USA, $55 \mathrm{mg} / \mathrm{kg}$ body weight, i. p., in $0.2 \mathrm{ml}$ of $10 \mathrm{mmol} / \mathrm{l}$ citrate buffer, pH 5.5) to animals fasted overnight. Blood samples for measurements of glucose were taken from the tail vein about $48 \mathrm{~h}$ after the streptozotocin injection and the day before they were killed. The rats with blood glucose of $13.9 \mathrm{mmol} / \mathrm{l}$ or more were considered as diabetic. The experimental groups comprised control and 3-week-old diabetic rats treated with or without SDI-157 (Hoechst Marion Roussel, $100 \mathrm{mg} \cdot \mathrm{kg}$ body weight $^{-1} \cdot$ day $^{-1}$, in the drinking water). The treatment of diabetic rats was started about $48 \mathrm{~h}$ after streptozotocin injection.

Reagents. Unless otherwise stated, all chemicals were of reagent-grade quality and were purchased from Sigma Chemical, St. Louis, Mo., USA. Methanol (HPLC grade), perchloric acid, hydrochloric acid, and sodium hydroxide were purchased from Fisher Scientific, Pittsburgh, Pa., USA. Ethyl alcohol (200 proof dehydrated alcohol, U.S.P. punctilious) was purchased from Quantum Chemical Co., Tiscola, Ill., USA. $\beta$-D-Glucose, sorbitol, N.F., myo-inositol, C.P., and D-fructose, U.S.P. were purchased from Pfanstiehl Laboratories (Waukegan, Ill., USA). Kits for malondialdehyde and 4-hydroxyalkenals assay were purchased from Oxis International (Portland, Ore., USA).

Experimental procedure. Rats from each group were sedated with carbon dioxide and subsequently killed by cervical dislocation. The femoral segments of the left sciatic nerve from each rat were rapidly ( $\sim 30 \mathrm{~s})$ dissected, carefully blotted with fine filter paper to remove any accompanying blood and frozen in liquid nitrogen for subsequent measurements of $\beta$-hydroxybutyrate, acetoacetate, glutamate, $\alpha$-ketoglutarate, ammonia, phosphocreatine, creatine and ATP. The remaining part of the left nerve and the right nerve were used for measurements of total malondialdehyde plus 4-hydroxyalkenals, reduced glutathione and sorbitol pathway intermediates.

It has been suggested (D. R. Tomlinson, personal communication) that euthanasia with prior sedation by carbon dioxide can cause respiratory acidosis and thus lead to a distorted nerve metabolite profile. This point was examined in a pilot experiment in which non-diabetic rats were killed either by decapitation without any anaesthesia $(n=10)$ or by cervical dislocation after prior sedation with $\mathrm{CO}_{2}(n=8)$. The rats were exposed to carbon dioxide $(\sim 15-20 \mathrm{~s})$ in a specially designed 
plastic chamber with two compartments. The lower compartment contained dry ice (the source of $\mathrm{CO}_{2}$ ) and was separated from the upper compartment (used as a rat container) by a septum perforated with numerous small holes. There was no difference found in the sciatic nerve reduced metabolite and high-energy phosphate concentrations between $\mathrm{CO}_{2}$-sedated and decapitated groups. Concentrations (in $\mu \mathrm{mol} / \mathrm{g}$ wet weight) of lactate were $1.49 \pm 0.39$ and $1.86 \pm 0.43$, pyruvate: $0.167 \pm 0.018$ and $0.142 \pm 0.024$, malate: $0.118 \pm 0.030$ and $0.131 \pm 0.043$, phosphocreatine: $2.86 \pm 0.36$ and $2.45 \pm 0.44$, and ATP: $0.902 \pm 0.227$ and $0.840 \pm 0.272$, respectively. No difference was found in free cytosolic $\mathrm{NAD}^{+}: \mathrm{NADH}$ ratios $(1054 \pm 202$ and $871 \pm 235)$, calculated as below. A tendency towards an increase in reduced metabolite concentrations and decrease in phosphocreatine and ATP concentrations and free cytosolic $\mathrm{NAD}^{+}: \mathrm{NADH}$ ratio in the decapitated compared with the $\mathrm{CO}_{2}$-sedated group can be explained by difficulties in dissecting sciatic nerves immediately after decapitation because of seizures of the body. The sampling time, critical for metabolites of dehydrogenase reactions as well as high-energy phosphates, was about $20 \mathrm{~s}$ longer for the decapitated than for the $\mathrm{CO}_{2}$-sedated group.

Measurements of sorbitol pathway intermediates and total malondialdehyde plus 4-hydroxyalkenals. Nerve segments $(\sim 40 \mathrm{mg})$ were weighed and homogenized in $1 \mathrm{ml} 0.1 \mathrm{~mol} / \mathrm{l} \mathrm{so}-$ dium-phosphate buffer, $\mathrm{pH}$ 6.5. A volume of $0.1 \mathrm{ml}$ of $0.3 \mathrm{~mol} / 1$ zinc sulphate, followed by an equivalent of barium hydroxide, was then added to $0.4 \mathrm{ml}$ of the homogenate for protein precipitation. The samples were centrifuged at $4000 \times g$ for $10 \mathrm{~min}$ (Sorvall MC 12V, Newton, Conn., USA) and aliquots of the supernatant were taken for spectrofluorometric measurements of glucose, sorbitol and fructose by enzymatic procedures as described previously [46, 47]. The rest of the homogenate was centifuged at $3000 \times g$ for $5 \mathrm{~min}$. Then $0.2 \mathrm{ml}$ of supernatant was used for measurements of total malondialdehyde plus 4hydroxyalkenals using LPO-586 kits from Oxis International (Portland, Ore., USA). The method [48] is based on the reaction of a chromogenic reagent, $N$-methyl-2-phenylindole with malondialdehyde and 4-hydroxyalkenals, after their extraction under methanesulphonic acidic conditions, at $45^{\circ} \mathrm{C}$. The absorbance of chromogenic product was measured at $586 \mathrm{~nm}$ using a spectrophotometer Beckman DU 640 (Fullerton, Calif., USA) and was compared with the absorbance in corresponding standards.

Preparation of perchloric extract. Femoral segments $(\sim 20 \mathrm{mg})$ of the left nerve as well as segments $(\sim 20 \mathrm{mg})$ of a remaining part of the left nerve or the right nerve were weighed, homogenized in $1.5 \mathrm{ml}$ of ice-cold $6 \% \mathrm{HCIO}_{4}$ and centrifuged at $4000 \times g$ for $10 \mathrm{~min}$. After centrifugation the samples were immediately neutralized with $5 \mathrm{~mol} / \mathrm{K} \mathrm{K}_{2} \mathrm{CO}_{3}$ to $\mathrm{pH}$ 6-7 and were centrifuged again at $4000 \times g$ for 5 min to precipitate insoluble $\mathrm{KClO}_{4}$.

Measurements of glycolytic and tricarboxylic acid cycle intermediates, ketone bodies, glutamate, ammonia, high-energy phosphates and creatine. The steady-state concentrations of acetoacetate, $\beta$-hydroxybutyrate, glutamate, $\alpha$-ketoglutarate, ammonia, lactate, pyruvate, phosphocreatine, creatine and ATP were assayed in perchloric extracts of femoral segments of the left sciatic nerve spectrofluorometrically (Perkin-Elmer LS-5B, Norwalk, Conn., USA) by enzymatic procedures as described [49]. The lower limit for all spectrofluorometric procedures in our study including glucose, sorbitol and fructose was $0.1 \times 10^{-9} \mathrm{~mol} / \mathrm{l}$.
GSH measurements. We modified a reported method [50] and mixed $0.1 \mathrm{ml}$ of neuralized nerve perchloric extracts with $0.89 \mathrm{ml}$ of $20 \mathrm{mmol} / \mathrm{l}$ EDTA in $1.0 \mathrm{~mol} / \mathrm{l} \mathrm{TRIS}-\mathrm{HCl}$ buffer $(\mathrm{pH}$ 8.1 ). The reaction was initiated by addition of $0.01 \mathrm{ml}$ of 0 phthaldialdehyde (10 mg-1 ml methanol). Initial and final readings were taken at $\lambda$ excitation: $345 \mathrm{~nm}, \lambda$ emission: 425 $\mathrm{nm}$, slits: 5 and 5 . The differences in initial and final readings were compared with those in corresponding GSH standards $\left(1-10 \times 10^{-9} \mathrm{M}\right)$ processed in the same run.

Calculations of free mitochondrial and cytosolic $N A D^{+}: N A D H$ ratios. According to classical publications of Krebs' laboratory $[51,52]$ and other studies [53], direct measurements of NAD, $\mathrm{NADH}, \mathrm{NADP}$ and NADPH do not provide information on compartmentalization of nicotinamide adenine nucleotides between cytosol and mitochondria and do not separate free from protein-bound forms (only free forms determine direction and free-energy changes of dehydrogenase reactions). The same studies proposed an alternative approach for assessment of free NAD $(\mathrm{P})^{+}: \mathrm{NAD}(\mathrm{P}) \mathrm{H}$ ratios in the cytoplasm and mitochondria from ratios of the concentrations of oxidized and reduced metabolites of suitable NAD(P)-linked dehydrogenase systems. Using this approach, free $\mathrm{NAD}^{+}: \mathrm{NADH}$ ratios for mitochondrial cristae, matrix as well as cytoplasm were calculated from the steady-state metabolite concentrations and the equilibrium constants of $\beta$-hydroxybutyrate, glutamate and lactate dehydrogenase systems as described [51, 53]:

$\frac{\left[\mathrm{NAD}^{+}\right]}{[\mathrm{NADH}]}$ mit cristae $=\frac{[\text { Acetoacetate }]}{[\beta \text {-Hydroxybutyrate }]} \times \frac{1}{\mathrm{k}_{1}}$,

where $\mathrm{k}_{1}$ is the equilibrium constant of $\beta$-hydroxybutyrate dehydrogenase.

$\frac{\left[\mathrm{NAD}^{+}\right]}{[\mathrm{NADH}]}{ }_{\text {mit matrix }}=\frac{[\alpha-\text { Ketoglutarate }] \times[\text { Ammonia }]}{[\text { Glutamate }]} \times \frac{1}{\mathrm{k}_{2}}$,

where $\mathrm{k}_{2}$ is the equilibrium constant of glutamate dehydrogenase.

$\frac{\left[\mathrm{NAD}^{+}\right]}{[\mathrm{NADH}]}$ cytoplasm $=\frac{[\text { Pyruvate }]}{[\text { Lactate }]} \times \frac{1}{\mathrm{k}_{3}}$,

where $\mathrm{k}_{3}$ is the equilibrium constant of lactate dehydrogenase

Statistical analysis. The results are expressed as means \pm standard deviation. Differences among experimental groups were evaluated by ANOVA, and the significance of differences between these groups assessed by the Student-Newman-Keuls multiple range test. Significance was defined at $p 0.05$ or less.

\section{Results}

Although the initial body weights were similar the final body weights were lower in diabetic rats than in control rats (Table 1). No significant difference was found between body weights in control and diabetic rats treated with SDI and the corresponding untreated groups.

Blood glucose concentrations were increased about 4.8-fold in diabetic rats compared with those in control rats. Treatment with SDI had no effect on blood glucose concentrations in either control or diabetic rats.

Sciatic nerve glucose concentrations were 3.6-fold higher in diabetic rats compared with those in control 
Table 1. Final body weights $(\mathrm{g})$ and blood glucose fructose concentrations $(\mathrm{mmol} / \mathrm{l})$ in control and diabetic rats treated with or without SDI $(n=7-9)$

\begin{tabular}{lrrrr}
\hline & Control & Control + SDI & Diabetic & Diabetic + SDI \\
\hline Body weight & $384.5 \pm 24.3$ & $360.4 \pm 28.9$ & $307.8 \pm 27.2^{\mathrm{a}}$ & $315.6 \pm 30.4^{\mathrm{a}}$ \\
Blood glucose & $4.08 \pm 0.60$ & $4.30 \pm 0.73$ & $19.52 \pm 3.65^{\mathrm{a}}$ & $18.36 \pm 4.04^{\mathrm{a}}$ \\
\hline
\end{tabular}

a Significantly different compared with those in controls $(p<0.01)$

Table 2. Nerve glucose, sorbitol and fructose concentrations ( $\mu \mathrm{mol} / \mathrm{g}$ wet weight) in control and diabetic rats treated with or without SDI $(n=7-9)$

\begin{tabular}{lllrr}
\hline & Control & Control + SDI & Diabetic & Diabetic + SDI \\
\hline Glucose & $3.24 \pm 0.94$ & $2.89 \pm 0.83$ & $11.78 \pm 4.17^{\mathrm{b}}$ & $11.32 \pm 2.78^{\mathrm{b}}$ \\
Sorbitol & $0.149 \pm 0.034$ & $1.63 \pm 0.29^{\mathrm{b}}$ & $1.65 \pm 0.36^{\mathrm{b}}$ & $7.34 \pm 1.77^{\mathrm{b}, \mathrm{c}}$ \\
Fructose & $1.22 \pm 0.15$ & $0.57 \pm 0.093^{\mathrm{b}}$ & $6.66 \pm 2.10^{\mathrm{b}}$ & $1.73 \pm 0.77^{\mathrm{a}, \mathrm{c}}$ \\
\hline
\end{tabular}

a,b Significantly different compared with those in controls $(p<0.05$ and $p<0.01$, respectively).

c Significantly different compared with those in untreated diabetics $(p<0.01)$

Table 3. Steady-state concentrations ( $\mu \mathrm{mol} / \mathrm{g}$ wet weight) of metabolites of $\beta$-hydroxybutyrate dehydrogenase, glutamate dehydrogenase and lactate dehydrogenase systems in control and diabetic rats treated with or without SDI $(n=7-8)$

\begin{tabular}{lcccc}
\hline & Control & Control + SDI & Diabetic & Diabetic + SDI \\
\hline Acetoacetate & $0.099 \pm 0.021$ & $0.107 \pm 0.037$ & $0.447 \pm 0.169^{\mathrm{b}}$ & $0.486 \pm 0.086^{\mathrm{b}}$ \\
$\beta$-Hydroxybutyrate & $0.063 \pm 0.015$ & $0.059 \pm 0.010$ & $0.438 \pm 0.151^{\mathrm{b}}$ & $0.459 \pm 0.135^{\mathrm{b}}$ \\
Glutamate & $1.92 \pm 0.22$ & $1.77 \pm 0.25$ & $2.90 \pm 0.28^{\mathrm{b}}$ & $1.84 \pm 0.19^{\mathrm{d}}$ \\
$\alpha$-Ketoglutarate & $0.141 \pm 0.052$ & $0.135 \pm 0.017$ & $0.109 \pm 0.034$ & $0.085 \pm 0.017^{\mathrm{a}}$ \\
Ammonia & $1.30 \pm 0.106$ & $1.28 \pm 0.18$ & $1.63 \pm 0.21^{\mathrm{a}}$ & $1.25 \pm 0.34^{\mathrm{c}}$ \\
Lactate & $1.05 \pm 0.147$ & $1.13 \pm 0.22$ & $2.98 \pm 1.46^{\mathrm{b}}$ & $1.53 \pm 0.24^{\mathrm{d}}$ \\
Pyruvate & $0.158 \pm 0.034$ & $0.193 \pm 0.038$ & $0.211 \pm 0.072$ & $0.140 \pm 0.023^{\mathrm{c}}$ \\
\hline
\end{tabular}

a,b Significantly different compared with those in controls $(p<0.05$ and $p<0.01$, respectively).

c,d Significantly different compared with those in untreated diabetics $(p<0.05$ and $p<0.01$, respectively)

rats (Table 2). Treatment with SDI had no effect on nerve glucose concentrations in either control or diabetic rats. Sorbitol concentration was increased 10.9fold and fructose concentration reduced 2.1-fold in control rats treated with SDI compared with the untreated group. Concentrations of sorbitol and fructose were increased 11.1-fold and 5.5-fold by diabetes. Treatment with SDI caused a 4.4-fold increase in nerve sorbitol and 3.8-fold reduction in nerve fructose in diabetic rats.

Concentrations of acetoacetate, $\beta$-hydroxybutyrate, glutamate, $\alpha$-ketoglutarate, ammonia, lactate and pyruvate were similar in the sciatic nerves of control rats treated with and without SDI (Table 3). Concentrations of acetoacetate and $\beta$-hydroxybutyrate were increased 4.5-fold and sevenfold in diabetic rats compared with those in control rats and these variables were not affected by SDI teatment. Concentrations of glutamate and ammonia were increased 1.5-fold and 1.3-fold by diabetes and an increase of both variables was completely prevented by SDI treatment. Concentrations of $\alpha$-ketoglutarate tended to decrease in diabetes although the difference to the control group did not achieve statistical significance and were reduced in diabetic rats treated with SDI (1.7-fold vs control group). Lactate concentrations were increased 2.8-fold in diabetic rats compared with those in control rats and this increase was reduced by SDI treatment. No significant difference was found between pyruvate concentrations in control and untreated diabetic rats whereas pyruvate concentrations in diabetic rats treated with SDI appeared to be reduced compared with those in the untreated diabetic group $(p=0.05)$.

Free $\mathrm{NAD}^{+}: \mathrm{NADH}$ ratios in nerve mitochondrial cristae (Fig.1,A), matrix (Fig. 1,B) and cytosol (Fig. 1,C) were decreased 1.8-fold, 1.5-fold and twofold in diabetic rats compared with those in control rats. None of these variables was affected by SDI treatment in either control or diabetic rats.

Nerve ATP concentrations were similar in control and diabetic groups treated with and without SDI (Table 4). Phosphocreatine concentrations and phosphocreatine:creatine ratios were not different in control rats treated with and without SDI. Both phosphocreatine concentrations and phosphocreatine:creatine ratios were reduced in diabetic rats (1.3-fold and 1.7-fold vs control group) and the decrease in both variables was further exacerbated by SDI treatment (1.6-fold and 1.4-fold vs untreated diabetic group 

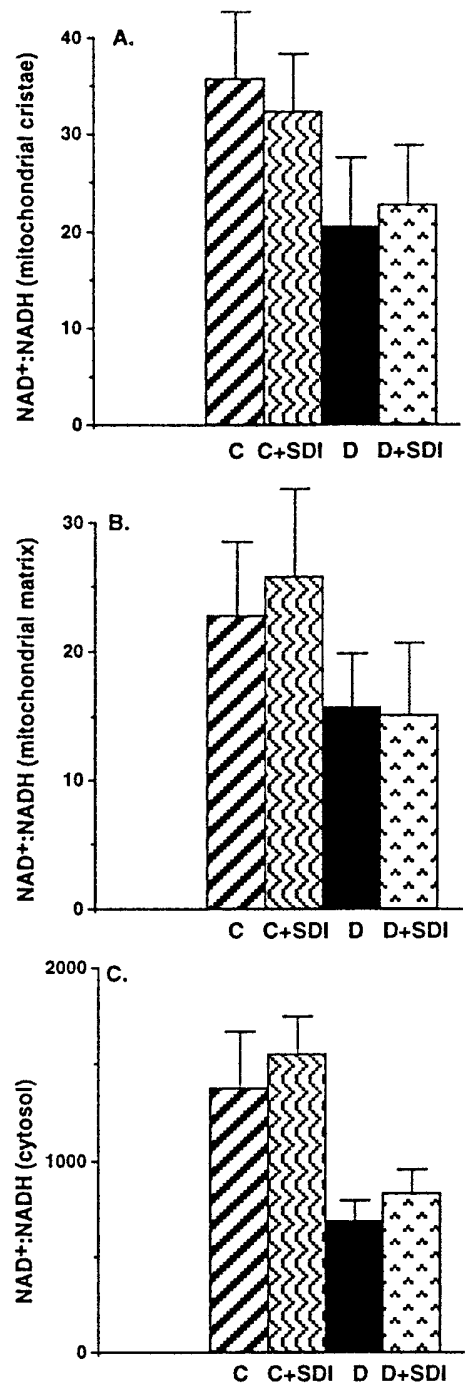

Fig.1A-C. Sciatic nerve free $\mathrm{NAD}^{+}: \mathrm{NADH}$ ratios in mitochondrial cristae (A), matrix (B) and cytosol (C) of control and diabetic rats treated with or without SDI $(n=7-8)$. C, control; D, diabetic

and 2.1-fold and 2.3-fold vs control group). Creatine concentrations were not different among control and diabetic rats treated with and without SDI.

Nerve total malondialdehyde plus 4-hydroxyalkenals (Fig. 2A) and GSH concentrations (Fig.2,B) were similar in control rats treated with and without

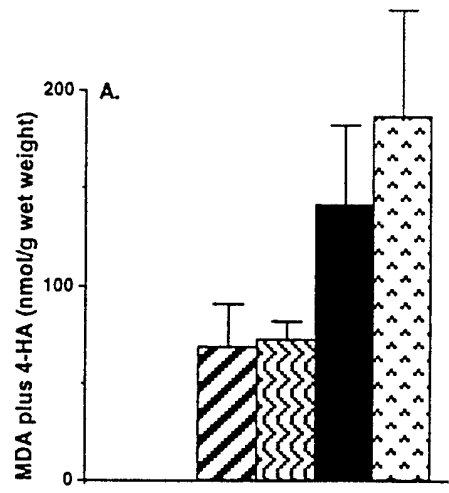

C $\mathrm{C}_{+} \mathrm{SOI} \mathrm{D} \mathrm{D}+\mathrm{SDI}$

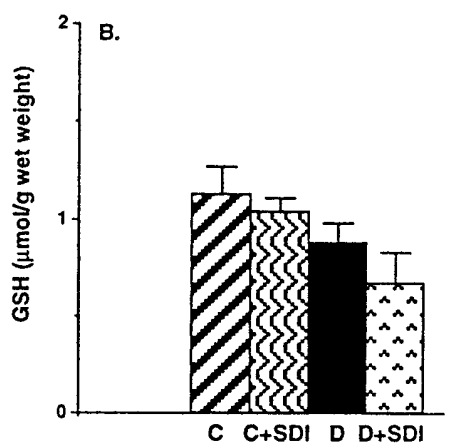

Fig. 2 A, B. Sciatic nerve malondialdehyde (MDA) plus 4-hydroxyalkenals (4-HA) (A) and GSH (B) concentrations in control and diabetic rats treated with or without SDI $(n=7-17)$. C, control; D, diabetic

SDI. Total malondialdehyde plus 4-hydroxyalkenals concentrations were increased whereas GSH concentrations were reduced in diabetic rats compared with control rats ( $p<0.01$ for both comparisons). The increase in total malondialdehyde plus 4-hydroxyalkenals and the reduction in GSH were further exacerbated by SDI treatment ( $p<0.05$ and $p<0.01$ vs untreated diabetic group, respectively).

\section{Discussion}

Our study shows that diabetes causes a considerable reduction in the free $\mathrm{NAD}^{+}: \mathrm{NADH}$ ratio, i.e. a shift towards a more reduced state of free mitochondrial NAD-couple, in the nerve mitochondrial cristae

Table 4. Nerve energy status in control and diabetic rats treated with or without SDI $(n=7-8)$

\begin{tabular}{lcccc}
\hline & Control & Control + SDI & Diabetic & Diabetic + SDI \\
\hline ATP & $0.832 \pm 0.272$ & $0.778 \pm 0.218$ & $0.674 \pm 0.226$ & $0.693 \pm 0.112$ \\
PCr & $3.14 \pm 0.461$ & $2.83 \pm 0.55$ & $2.42 \pm 0.38^{\mathrm{a}}$ & $1.52 \pm 0.39^{\mathrm{b}, \mathrm{d}}$ \\
Creatine & $4.70 \pm 0.63$ & $4.40 \pm 0.96$ & $5.45 \pm 1.38$ & $5.14 \pm 1.29$ \\
PCr/Creatine & $0.689 \pm 0.165$ & $0.649 \pm 0.045$ & $0.413 \pm 0.076^{\mathrm{b}}$ & $0.301 \pm 0.062^{\mathrm{b}, \mathrm{c}}$ \\
\hline
\end{tabular}

PCr, phosphocreatine. Concentrations of ATP, PCr and Creatine are expressed in $\mu \mathrm{mol} / \mathrm{g}$ wet weight.

a,b Significantly different compared with those in controls $(p<0.05$ and $p<0.01$, respectively). c,d Significantly different compared with those in untreated diabetics $(p<0.05$ and $p<0.01$, respectively) 
which can be regarded as metabolic evidence of endoneurial hypoxia. This is consistent with findings of decreased blood flow in both exposed $[4,54-58]$ and unexposed [59] diabetic nerve. The shifts in free $\mathrm{NAD}^{+}: \mathrm{NADH}$ ratios in mitochondrial matrix and cristae appear unidirectional. This is predictable considering the dependence of NADH-generating capacity of the tricarboxylic acid cycle, localized in mitochondrial matrix, on the efficacy of NADH oxidation by the respiratory chain in mitochondrial cristae [60]. The absence of any improvement in free $\mathrm{NAD}^{+}: \mathrm{NADH}$ ratios in nerve mitochondrial matrix and cristae in the diabetic rats treated with SDI compared with the untreated diabetic group is consistent with a previous report of the lack of any effect of this compound on nerve blood flow [4].

In spite of the effective inhibition of SDH in the diabetic peripheral nerve with the applied dose of SDI (consistent with the study [30]) we did not observe any increase in free cytosolic $\mathrm{NAD}^{+}: \mathrm{NADH}$ ratio. This does not agree with the report [29] of decreased NAD-redox imbalances (expressed as lactate:pyruvate ratios) in incubated endoneurial preparations from diabetic rats treated with SDI. Our results differ from those [29] despite the the use of similar (prevention) design, similar duration of diabetes and a more profound inhibition of SDH in our experiments. Our findings do not support the key role of SDH in cytosolic NAD-redox imbalances in the diabetic nerve. The decrease induced by diabetes in the nerve-free cytosolic $\mathrm{NAD}^{+}: \mathrm{NADH}$ ratio is probably of mitochondrial origin, because cytoplasmic and mitochondrial pools of nicotinamide nucleotides are linked through dicarboxylate carriers and the nerve strongly depends on aerobic metabolism [42, 61].

The changes in high-energy phosphates induced by diabetes in our study are consistent with previous reports $[42,43]$. Note that SDI treatment exacerbated both phosphocreatine deficit and decrease in the phosphocreatine/creatine ratio, the most sensitive variable of neural tissue energy status $[62,63]$ in the diabetic peripheral nerve. Although this phenomenon requires specific studies, at least two putitative underlying mechanisms can be invoked. Excessive sorbitol accumulation in the peripheral nerve of rats treated with SDI can potentially cause (through $\mathrm{Ca}^{++}$ or another mediator) uncoupling of mitochondrial oxidation and phosphorylation and thus lead to a depletion of phosphocreatine, the storage form of highenergy phosphates. That an osmotic factor is involved is supported by decreased phosphocreatine concentrations in the peripheral nerve of rats after 10 days of feeding with $50 \%$ galactose but not in rats which have been diabetic for 10 days (nerve galactitol accumulation in the galactose-fed rats exceeded $\sim 13$-fold nerve sorbitol accumulation in the diabetic group) (I. Obrosova, unpublished observations). It is also supported by experiments with DL- $\alpha$-lipoic acid which increased nerve sorbitol accumulation [44, 64] and did not prevent decrease in either phosphocreatine concentrations [64] or the phosphocreatine:creatine ratio [44], in spite of the preservation of normal blood flow [16, 44] and free mitochondrial NAD: NADH ratios [44], in the "young"[65] streptozotocin model of diabetes. Another explanation for exacerbation of energy deficiency induced in diabetes by SDI is the possibility that the sorbitol pathway is the route providing fructose-6-phosphate for anaerobic glycolysis [66], bypassing hexokinase. Aerobic metabolism of the diabetic peripheral nerve is depressed due to hypoxia and activated glycolysis represents an important compensatory mechanism of high-energy phosphate production [42, 61]. Hexokinase has a high affinity for glucose [67] and is easily saturated [68] thus becoming the rate-limiting step for the glycolytic flux. The peripheral nerve can use fructose as an alternative source of energy [69] and the presence of fructose-1-phosphate, which can be formed from fructose and isomerized to fructose-6-phosphate, in the nerve has been documented [70]. Thus, the inhibition of SDH in diabetes eliminates the potentially important source of glycolytic intermediates which, due to inhibition of glycolysis, would ultimately result in energy failure. Although the provision of fructose-6phosphate by the sorbitol pathway would also be blocked by aldose reductase inhibitors, the latter is unimportant for the diabetic nerve energy status as aldose reductase inhibition ameliorates nerve blood flow $[4,71]$ and eliminates endoneurial hypoxia [71] thus improving aerobic glucose oxidation providing much greater energy yield than the anaerobic glycolysis [72].

Nerve GSH concentration obtained with $O$-phthaldialdehyde assay in our experiments is in agreement with studies which have used HPLC [16] and the colorimetric procedure with Ellman's reagent [73]. Decrease of nerve GSH concentration induced by diabetes is consistent with a previous report [16] and parallels accumulation of malondialdehyde plus 4-hydroxyalkenals, the products of lipid peroxidation. Exacerbation of both lipid aldehyde accumulation and GSH depletion induced by diabetes by SDI treatment as well as the lack of any prooxidant properties of the SDI-157 in itself (manifested by the absence of lipid aldehyde accumulation and GSH depletion in non-diabetic rats treated with SDI compared with an untreated group), indicates that SDI treatment enhances indirectly oxidative stress induced by diabetes.

In conclusion, our findings do not support the concept of "diabetic pseudohypoxia", and imply that minor flux through SDH can not account for the changes in such a dynamic system as the redox state of nicotinamide dinucleotides, in the diabetic nerve. Our results are consistent with observations of other groups showing that SDH inhibition does not offer 
an effective approach for treatment of functional [4] and metabolic [12] deficits characteristic for experimental diabetic neuropathy. Moreover, exacerbation of energy deficiency and oxidative stress induced by SDI indicates that SDH inhibition in the peripheral nerve in diabetes is adverse rather than beneficial and is in agreement with the findings of a large increase in neuroaxonal dystrophy [41] rather than with reports of beneficial effects of SDIs.

\section{References}

1. Gillon KR, Hawthorne JN, Tomlinson DR (1983) Myo-inositol and sorbitol metabolism in relation to peripheral nerve function in experimental diabetes in the rat: the effect of aldose reductase inhibition. Diabetologia 25: 365-371

2. Calcutt NA, Mizisin AP, Kalichman MW (1994) Aldose reductase inhibition, Doppler flux and conduction in diabetic rat nerve. Eur J Pharmacol 251: 27-33

3. Hotta N, Koh N, Sakakibara F et al. (1996) Effect of propionylL-carnitine on motor nerve conduction, autonomic cardiac function, and nerve blood flow in rats with streptozotocin-induced diabetes: comparison with an aldose reductase inhibitor. J Pharmacol Exp Ther 276: 49-55

4. Cameron NE, Cotter MA, Basso M, Hohman TC (1997) Comparison of the effects of inhibitors of aldose reductase and sorbitol dehydrogenase on neurovascular function, nerve conduction, and tissue polyol pathway metabolites in streptozotocindiabetic rats. Diabetologia 40: 271-281

5. Ino-ue $M$, Yokogawa $H$, Yamamoto $M$, Naka $H$, Kuriyama $H$ (1998) Structural impairments in optic nerve of diabetic rats ameliorated with the aldose reductase inhibitor. Exp Eye Res 66: 397-401

6. Ziegler D, Mayer P, Rathwann W, Gries FA (1991) One-year treatment with the aldose reductase inhibitor, ponalrestat, in diabetic neuropathy. Diabetes Res Clin Pract 14: 63-73

7. Hotta N, Sakamoto N, Shigeta Y, Kikkawa R, Goto Y (1996) Clinical investigation of epalrestat, an aldose reductase inhibitor, on diabetic neuropathy in Japan: multicenter study. Diabetic Neuropathy Study Group in Japan. J Diabetes Complications 10: 168-172

8. Pfeifer MA, Schumer MP, Gelber DA (1997) Aldose reductase inhibitors: the end of an era or the need for different trial designs? Diabetes 46 [Suppl 2]:S82-S89

9. Greene DA, Arezzo JCA, Brown MB (1999) Effect of aldose reductase inhibition on nerve conduction and morphometry in diabetic neuropathy. Neurology (in press)

10. Kinoshita JH, Nishimura C (1988) The involvement of aldose reductase in diabetic complications. Diabetes Metab Rev 4: 323-337

11. Kador PF (1988) The role of aldose reductase in the development of diabetic complications. Med Res Rev 8: 325-352

12. Kuruvilla R, Eichberg J (1998) Depletion of phospholipid arachidonoyl-containing molecular species in a human Schwann cell line grown in elevated glucose and their restoration by an aldose reductase inhibitor. J Neurochem 71: 775-783

13. Fernyhough P, Brewster WJ, Fernandes K, Diemel LT, Tomlinson DR (1998) Stimulation of nerve growth-factor and substance $\mathrm{P}$ expression in the iris-trigeminal axis of diabetic rats involvement of oxidative stress and effects of aldose reductase inhibition. Brain Res 802: 247-253

14. Ohi T, Saita K, Furikawa S, Ohta M, Hayashi K, Matsukura S (1998) Therapeutic effects of aldose reductase inhibitor on experimental diabetic neuropathy through synthesis/secretion of nerve growth factor. Exp Neurol 151: 215-220

15. Cameron NE, Cotter MA, Archibald V, Dines KC, Maxfield EK (1994) Anti-oxidant and pro-oxidant effects on nerve con- duction velocity, endoneurial blood flow and oxygen tension in non-diabetic and streptozotocin-diabetic rats. Diabetologia 37: 449-459

16. Nagamatsu M, Nickander KK, Schmelzer JD et al. (1995) Lipoic acid improves nerve blood flow, reduces oxidative stress, and improves distal nerve conduction in experimental diabetic neuropathy. Diabetes Care 18: 1160-1167

17. Cotter MA, Love A, Watt MJ, Cameron NE, Dines KC (1995) Effects of natural free radical scavengers on peripheral nerve and neurovascular function in diabetic rats. Diabetologia 38 : $1285-1294$

18. Sugimoto K, Yagihashi S (1997) Effects of aminoguanidine on structural alterations of microvessels in peripheral nerve of streptozotocin diabetic rats. Microvasc Res 53: 105-112

19. Low PA, Nickander KK, Trischler HJ (1997) The Roles of Oxidative Stress and Antioxidant Treatment in Experimental Diabetic Neuropathy. Diabetes 46 [Suppl 2]: S38-S42

20. Cameron NE, Cotter MA (1997) Metabolic and vascular factors in the pathogenesis of diabetic neuropathy. Diabetes 46 [Suppl 2]:S31-S37

21. Greene DA, Lattimer S, Ulbrecht J, Carroll P (1985) Glucoseinduced alterations in nerve metabolism: Current perspective on the pathogenesis of diabetic neuropathy and future directions for research and therapy. Diabetes Care 8: 290-299

22. Zhu X, Eichberg J (1993) Molecular species composition of glycerolipids in rat sciatic nerve and its alteration in streptozotocin-induced diabetes. Biochim Biophys Acta 1168: 1-12

23. Sozuki E, Yasuda K, Yasuda K et al. (1994) 1H-NMR analysis of nerve edema in the streptozotocin-induced diabetic rat. J Lab Clin Med 124: 627-637

24. Doss DJ, Kuruvilla R, Bianchi R, Peterson RG, Eichberg J (1997) Effects of hypoxia and severity of diabetes on Na, KATPase activity and arachidonoyl-containing molecular species in streptozotocin-diabetic rat nerve. J Peripheral Nerv Syst 2: $1-9$

25. Mizisin AP, Bache M, DiStefano PS, Acheson A, Lindsay RM, Calcutt NA (1997) BDNF attenuates functional and structural disorders in nerves of galactose-fed rats. J Neuropath Exp Neurol 56: 1290-1301

26. Hounsom L, Horrobin DF, Tritschler H, Corder R, Tomlinson DR (1998) A lipoic acid-gamma linolenic acid conjugate is effective against multiple indices of experimental diabetic neuropathy. Diabetologia 41: 839-844

27. Apfel SC, Kessler JA, Adornato BT, Litchy WJ, Sanders C, Rask CA (1998) Recombinant human nerve growth factor in the treatment of diabetic polyneuropathy. NGF Study Group. Neurology 51: 695-702

28. Williamson JR, Chang K, Frangos M et al. (1993) Hyperglycemic pseudohypoxia and diabetic complications. Diabetes 42: 801-813

29. Tilton RG, Chang K, Neyengaard JR, van den Enden M, Ido Y, Williamson JR (1995) Inhibition of sorbitol dehydrogenase. Effects on vascular and neural dysfunction in streptozotocininduced diabetic rats. Diabetes 44: 234-242

30. Geissen K, Utz R, Grotsch H, Lang J, Nimmesgern H (1994) Sorbitol-accumulating Pyrimidine Derivatives. Arzneimittelforschung 44: 1032-1043

31. Oates P, Schelhorn T, Miller M et al. (1998) Polyol pathway inhibitors dose-dependently preserve nerve function in diabetic rats. Diabetologia 41 [Suppl 1]:A271 (Abstract)

32. Ng DT, Lee FK, Song ZT et al. (1998) Effects of sorbitol dehydrogenase deficiency on nerve conduction in experimental diabetic mice. Diabetes 47: 961-966

33. Delcroix JD, Michael GJ, Priestley JV, Tomlinson DR, Fernyhough P (1998) Effect of nerve growth factor treatment on p75NTR gene expression in lumbar dorsal root ganglia of streptozotocin-induced diabetic rats. Diabetes 47: $1779-1785$

34. Obrosova I, Kuchmerovskaya T, Parkhomets P, Klimenko A, Yefimov A (1994) Serotonin and GABA uptake and release by brain cortex synaptosomes of streptozotocin-diabetic and 
galactosemic rats: effect of aldose reductase inhibitor. Diabetologia Croatica 23: 63-70

35. Ralevic V, Belai A, Burnstock G (1995) Effects of streptozotocin-diabetes on sympathetic nerve, endothelial and smooth muscle function in the rat mesenteric arterial bed. Eur J Pharmacol 286: 193-199

36. Kamei J, Ohsawa M (1996) Effects of diabetes on methamphetamine-induced preference in mice. Eur J Pharmacol 318: 251-256

37. Mizisin AP, Shelton GD, Wagner S, Rusbridge C, Powell HC (1998) Myelin splitting, Schwann cell injury and demyelination in feline diabetic neuropathy. Acta Neuropathol (Berl) 95: 171-174

38. Kalichman MW, Powell HC, Mizisin AP (1998) Reactive, degenerative, and proliferative Schwann cell responses in experimental galactose and human diabetic neuropathy. Acta Neuropathol (Berl) 95: 47-56

39. Chokroverty S, Seiden D, Navidad P, Cody R (1988) Distal axonopathy in streptozotocin diabetes in rats. Experientia 44: 444-446

40. Fernyhough P, Gallagher A, Averill SA et al. (1999) Aberrant neurofilament phosphorylation in sensory neurons of rats with diabetic neuropathy. Diabetes 48: 881-889

41. Schmidt RE, Dorsey DA, Beaudet LN, Plurad SB, Williamson JR, Ido Y (1998) Effect of sorbitol dehydrogenase inhibition on experimental diabetic autonomic neuropathy. J Neuropath Exp Neurol 57: 1175-1189

42. Low PA, Ward K, Schmelzer JD, Brimijoin S (1985) Ischemic conduction failure and energy metabolism in experimental diabetic neuropathy. Am J Physiol 248: E457-E462

43. Low PA, Schmelzer JD, Ward KK, Curran GL, Poduslo JF (1988) Effect of hyperbaric oxygenation on normal and chronic streptozotocin diabetic peripheral nerves. Exp Neurol 99: 201-212

44. Greene DA, Cao X, Van Huysen C, Obrosova IG (1998) Effects of DL- $\alpha$-lipoic acid (LA) on diabetic nerve function, bioenergetics, and antioxidative defense. Diabetes 47 [Suppl 1]:A136 (Abstract)

45. Bravenboer B, Kappelle AC, Hamers FP, van Buren T, Erkelens DW, Gispen WH (1992) Potential use of glutathione for the prevention and treatment of diabetic neuropathy in the streptozotocin-induced diabetic rat. Diabetologia 35: 813-814

46. Obrosova I, Cao X, Greene DA, Stevens MJ (1998) Diabetesinduced changes in lens antioxidant status, glucose utilization and energy metabolism: effect of DL- $\alpha$-lipoic acid. Diabetologia 41: 1442-1450

47. Obrosova IG, Stevens MJ (1999) Effect of dietary taurine supplementation on GSH and NAD(P)-redox status, lipid peroxidation, and energy metabolism in diabetic precataractous lens. Invest Ophthalmol Vis Sci 40: 680-688

48. Erdelmeier I, Gerard-Monnier D, Yadan JC, Chaudiere J (1998) Reactions of N-methyl-2-phenylindole with malondialdehyde and 4-hydroxyalkenals. Mechanistic aspects of the colorimetric assay of lipid peroxidation. Chem Res Toxicol 11: 1184-1194

49. Lowry OH, Passoneau JV (1972) A Flexible System of Enzymatic Analysis. Academic Press, Orlando

50. Hissin PJ, Hilf R (1976) A fluorometric method for determination of oxidized and reduced glutathione in tissues. Anal Biochem 74: 214-226

51. Williamson DH, Lund P, Krebs HA (1967) The Redox State of Free Nicotinamide-Adenine Dinucleotide in the Cytoplasm and Mitochondria of Rat Liver. Biochem J 103: 514-527

52. Veech RL, Eggleston LV, Krebs HA (1969) The Redox State of Free Nicotinamide-Adenine Nucleotide Phosphate in the Cytoplasm of Rat Liver. Biochem J 115: 609-619

53. Masuda T, Dobson GP, Veech RL (1990) The Gibbs-Donnan Near-equilibrium System of Heart. J Biol Chem 265: 20321-20334
54. Tuck RR, Schmelzer JD, Low PA (1984) Endoneurial blood flow and oxygen tension in the sciatic nerves of rats with experimental diabetic neuropathy. Brain 107: 935-950

55. Hotta N, Koh N, Sakakibara F et al. (1996) Effects of beraprost sodium and insulin on the lectroretinogram, nerve conduction, and nerve blood flow in rats with streptozotocin-induced diabetes. Diabetes 45: 361-366

56. Dewhurst M, Omawari N, Tomlinson DR (1997) Aminoguanidine - effects on endoneurial vasoactive nitric oxide and on motor nerve conduction velocity in control anbd streptozotocin-diabetic rats. Br J Pharmacol 120: 593-598

57. Calcutt NA, Dines KC, Cesena RM (1998) Effects of the peptide HP228 on nerve disorders in diabetic rats. Metabolism 47: 650-656

58. Kalichman MW, Dines KC, Bobik M, Mizisin AP (1998) Nerve conduction velocity, laser Doppler flow, and axonal caliber in galactose and streptozotocin diabetes. Brain Res 810: 130-137

59. Sasaki H, Schmelzer JD, Zollman PJ, Low PA (1997) Neuropathology and blood flow of nerve, spinal roots and dorsal root ganglia in longstanding diabetic rats. Acta Neuropathol (Berl) 93: 118-128

60. Hassinen IE, Hiltunen K (1975) Respiratory control in isolated perfused rat heart. Role of the equilibrium reactions between the mitochondrial electron carriers and the adenylate system. Biochim Biophys Acta 408: 319-330

61. Low PA, Schmelzer JD, Ward KK, Yao JK (1986) Experimental chronic hypoxic neuropathy: relevance to diabetic neuropathy. Am J Physiol 250:E94-E99

62. Greene DA, Lattimer SA (1984) Impaired energy utilization and Na-K-ATP-ase in diabetic peripheral nerve. Am J Physiol 246: E311-E318

63. Ogawa S, Lee TM, Glynn P (1986) Energy metabolism in rat brain in vivo studied by $31 \mathrm{P}$ nuclear magnetic resonance: changes during postnatal development. Arch Biochem Biophys 248: $43-52$

64. Low PA, Yao JK, Kishi Y et al. (1997) Peripheral nerve energy metabolism in experimental diabetic neuropathy. Neurosci Res Commun 21: 49-56

65. Cameron NE, Cotter MA, Horrobin DH, Tritschler HJ (1998) Effects of alpha-lipoic acid on neurovascular function in diabetic rats: interaction with essential fatty acids. Diabetologia 41: 390-399

66. Cheng HM, Hirose K, Xiong H, Gonzalez RG (1989) Polyol pathway activity in streptozotocin-diabetic rats lens. Exp Eye Res 49: 87-92

67. Mehta A, Jarori GK, Kenkare UW (1988) Brain hexokinase has no pre-existing allosteric site for glucose 6-phosphate. J Biol Chem 263: 15492-15497

68. Vicario C, Arizmendi C, Malloch G, Clark JB, Medina JM (1991) Lactate utilization by isolated cells from early neonatal rat brain. J Neurochem 57: 1700-1707

69. Greene DA, Winegrad AI (1981) Effects of acute experimental diabetes on composite energy metabolism in peripheral nerve axons and Schwann cells. Diabetes 30: 967-974

70. Poulsom R, Mirrlees DJ, Earl DC, Heath H (1983) The effects of an aldose reductase inhibitor upon the sorbitol pathway, fructose-1-phosphate and lactate in the retina and nerve of streptozotocin-diabetic rats. Exp Eye Res 36: 751-760

71. Cameron NE, Cotter MA, Dines KC, Hohman TC (1996) Reversal of defective peripheral nerve conduction velocity, nutritive endoneurial blood flow, and oxygenation by a novel aldose reductase inhibitor, WAY-121, 509, in streptozotocin-induced diabetic rats. J Diabetes Complications 10: 43-53

72. Lehninger AL (1987) Principles of Biochemistry. Worth Publishers Inc., New York

73. Van Dam PS, Van Asbeck BS, Bravenboer B, Van Oirschot JF, Gispen WH, Marx JJ (1998) Nerve function and oxidative stress in diabetic and vitamin E-deficient rats. Free Radic Biol Med 24: 18-26 